\title{
LA CONTRIBUTION DU COMMENTAIRE À LA CONSTRUCTION DES SPECTATEURS ET DES TÉLÉSPECTATEURS DES MATCHES DE RUGBY
}

\author{
Valérie Bonnet et Robert Boure ${ }^{1}$
}

Avant d'être des événements et des cérémonies médiatiques destinés à un public élargi, la finale du Championnat de France de rugby et les matches du Tournoi des Cinq Nations étaient des événements et des rendez-vous sociaux ${ }^{2}$ pour le monde du rugby, schématiquement une petite communauté intriquée de pratiquants et de spectateurs initiés, très attachée aux dimensions identitaires et culturelles de ce sport (Darbon, 1999 ; Boure, Cassagne, 2007a ; Bonnet, Boure, 2008). L'une constituait le point d'orgue très attendu d'une saison qui ne s'animait vraiment qu'au sortir des poules, c'est-à-dire à partir des

1 Respectivement Maître de conférences et Professeur de Sciences de l'information et de la communication, LERASS (EA 827), Université Toulouse 3.

2 L'événement n'est pas seulement ce qui advient rituellement ou fortuitement dans une situation sociale donnée et qui fait date pour les acteurs concernés. C'est aussi ce qui perturbe un état de fait et crée une discontinuité ouvrant sur des possibles. C'est enfin ce qui est construit par les discours à propos de ce qui advient, de la discontinuité et des possibles, étant entendu que tous les acteurs ne sélectionnent pas les mêmes éléments dans ce qui arrive, ce qui est perturbé et ce qui pourrait poindre. Il est à l'interface de l'attendu et l'inattendu, de la surface et de la profondeur, du fait et du discours, du signifiant (il fait sens) et du significatif (il renvoie toujours à quelque chose). Sur la construction médiatique des événements, cf. notamment Véron, 1981 ; Dayan, Katz, 1992.

Recherches en communication, $\mathrm{n}^{\circ} 30$ (2008). 
matches à élimination directe; les autres étaient des rendez-vous rituels dont l'enjeu symbolique était, au-delà de l'étalonnage du XV de France par rapport aux équipes anglo-saxonnes, la suprématie entre les Nations de l'Hémisphère Nord, chaque sélection défendant les couleurs de son territoire national. Bien entendu, c'est à travers la communication que ces événements prenaient sens et que ce sens était partagé : ils constituaient des éléments forts du lien social entre aficionados et participaient, tant au travers des commentaires « à chaud », puis « à froid » ${ }^{1}$ des spectateurs, supporteurs et journalistes que des grands récits auxquels ils donnaient fréquemment lieu, à la construction d'une mémoire (plus que d'une histoire), voire d'une légende, avec ses épopées, ses exploits, ses drames, ses héros, ses équipes mythiques et ses stades emblématiques (sur les légendes sportives, cf. Barthes, 1956).

Progressivement, la nature de ces événements va se transformer sous le jeu complexe de causalités multiples liées à l'évolution des sociétés, de la place du sport et du spectacle sportif dans ces sociétés, des pratiques sportives et rugbystiques (en particulier la professionnalisation, effective dès 1995), des formes d'organisation des spectacles sportifs (l'événement est désormais préparé par l'événementiel dont la communication éponyme n'est que l'élément le plus voyant) et enfin des médias. Car l'ouverture du rugby français vers d'autres territoires que ses terroirs historiques du Sud de la Loire, d'autres publics, d'autres imaginaires, d'autres discours, d'autres pratiques, d'autres enjeux (économiques, symboliques...), commencée avec la presse écrite et continuée avec la radio qui introduit le temps réel du « direct », connaît une notable accélération à partir de 1957, quand la télévision publique retransmet les matches du Tournoi des Cinq Nations et la finale du Championnat de France. Dès lors, s'appuyant sur les progrès rapides de son implantation dans le paysage médiatique autant que sur l'élargissement tout aussi rapide de son audience et jouant sur les ressources des images et du commentaire ainsi que sur des dispositifs techniques de plus en plus sophistiqués, la télévision projette peu à peu dans les mêlées des publics plus nombreux et différents des publics des stades. Des publics que l'image et surtout le commentaire doivent, ente autres, éduquer à un sport et à une culture jusqu'ici confidentiels et relativement fermés... sans pour autant déplaire aux initiés qui, eux

1 Un match fait parler avant et après, en dehors du stade et des lieux de réception médiatique, a fortiori quand il constitue un événement. 
aussi, « regardent les matches à la télévision » (sur les fonctions du commentaire sportif, cf. Fernandez, 2004 ; Boure, Cassagne, 2007b).

Nous nous proposons, à partir de trois entrées, de mener une étude de la représentation des publics par la télévision lors d'événements rugbystiques de très niveau : la construction des publics dans la séquence d'avant-match d'abord, la place des publics dans les scènes et les dispositifs télévisuels ensuite, et enfin la mise en image et en son des dimensions festives de spectacles que d'aucuns ont pu qualifier de « cérémonies télévisuelles ». En référence au cinéaste Serge M. Eisenstein qui considérait que les rapports entre les parties d'un tout sont plus importants que chacune d'entre elles, nous traiterons chaque fois où cela sera possible ${ }^{1}$ les commentaires, et au-delà le son, en relation avec les images. Avec une hypothèse que nous empruntons à un autre spécialiste du cinéma, Michel Chion (1990, 10), selon lequel le son, et donc le commentaire, est «bien plus qu'une opinion plaquée sur une vision. C'est la structuration et l'orientation même de la vision qu'elle engage, en la cadrant rigoureusement ». Non seulement il fait voir les acteurs (joueurs, arbitres, spectateurs...), les actions de jeu ou le mouvement au-delà ce que la seule image permettrait de voir, mais encore il interprète ce que l'image montre, anticipe ce qu'elle montrera plus tard et met en rapport les images du match en train de se faire avec celles de matches passés (intertextualité).

Le corpus est composé, pour chaque décennie, de deux finales de championnat et d'un ou deux matches internationaux : France/Afrique du Sud, 1961 (F-AS/61) ; Dax/Mont de Marsan, 1963 (D-MdeM/63) ; Agen-Dax, 1966 (A-D/66) ; Ecosse/France, 1968 (E-F/68) ; Béziers/ Perpignan, 1977 (B-P/77) ; Béziers-Montferrand, 1978 (B-M/78) ; France/Nouvelle-Zélande, 1979 (F-NZ/79) ; France/Pays de Galles, 1983 (F-PG/83) ; Toulouse/Toulon, 1985 (T-To/85) ; Toulouse-Agen, 1986 (T-A/86) ; France/Nouvelle-Zélande, 1986 (F-NZ/86) ; ToulouseBrive, 1996 (T-Br/96) ; Toulouse-Bourgoin-Jallieu, 1997 (T-BJ/97) ; France-Nouvelle-Zélande, 1999 (F-NZ/99) ; Toulouse-Montferrand, 2001 (T-M/01) ; Agen-Biarritz, 2002 (A-Bi/02) ; France-Afrique

1 Notamment en raison de la taille de l'article demandée par la revue. C'est également pour cette raison que nous ne traiterons pas, au niveau de l'énonciation, les spécificités discursives tenant aux rôles dévolus au journaliste et au consultant ainsi qu'à l'équation personnelle, à la formation et aux méthodes de travail de chacun. 
du Sud, 2005 (F-AS/05). Tous ces matches (sauf France-NouvelleZélande, 1999, TF1), ont été retransmis par le service public de télévision dont on connaît les rapports partenariaux longtemps entretenus avec la Fédération Française de Rugby, situation qui n'est pas sans incidence sur l'énonciation du média et les conditions sociales de celle-ci (Bonnet, Boure, Cassagne, 2007).

\section{Les publics dans les commentaires d'avant-match}

La prise d'antenne d'un « direct » sportif est un moment important. Depuis quelques années, et cela n'est l'apanage ni du service public, ni des retransmissions des matches de rugby, l'antenne s'ouvre fréquemment sur un plan fixe (et américain) du journaliste et du consultant sur la toile de fond d'un stade où l'on perçoit à peine les spectateurs. Et c'est les yeux dans les yeux (Véron, 1983) qu'ils s'adressent aux téléspectateurs ${ }^{1}$, souvent à travers une formule standard du type «Bonjour mesdames, bonjour mesdemoiselles, bonjour messieurs », quelquefois complétée par un «bonjour amis sportifs », voire remplacée par un « Bonjour et bienvenue à tous » (F-NZ/86) ou encore un plus sobre «Bienvenue à tous » (A-Bi/02) rappelant que la retransmission s'adresse à un public plus large que les seuls rugbyphiles. Mais à la différence du présentateur du Journal Télévisé, « homme-tronc » et maître de cérémonie, ils n'ont pas la maîtrise complète du dispositif (elle est partagée avec le réalisateur) ${ }^{2}$ et c'est davantage par la voix que par le corps et

1 Ce type «d'adresse » est également une garantie pour les téléspectateurs que la retransmission est en direct. C'est entre autres pour cette raison qu'il revient à l'écran en plusieurs occasions : arrêts de jeu, mi-temps, fin du match. Cela étant, c'est essentiellement en voix off que les commentateurs se manifestent, autrement dit à travers une voix qui, selon les termes consacrés « fait voir l'image » (cf. infra).

2 La marge de manœuvre des commentateurs est toutefois importante. D'abord parce que le commentaire « à chaud » n'est pas toujours dépendant de l'image, ensuite parce qu'ils s'autorisent des initiatives dont la portée est loin d'être négligeable, cf. les propos de Roger Couderc (E-F/68) « Ce match je le dédie à la mémoire de deux grands joueurs français disparus tragiquement, Guy Boniface et Jean-Michel Capendeguy. D'ailleurs, c'est sur le programme officiel. La Scottish Rugby Union a tenu à rendre un hommage vibrant à nos deux disparus. Donc, c'est à eux que nous penserons en faisant ce reportage, et c'est à eux aussi que l'équipe de France dédie ce match, à leur souvenir, à leur mémoire ». Le journaliste décide donc de dédier le match aux « disparus », et relaie la dédicace de l'équipe de France aux deux joueurs victimes d'un accident. Il peut d'autant plus revendiquer cette posture que sa légitimité rugbystique est assurée : en tant que journaliste-supporteur, il est le 
l'image qu'ils imposent leur présence et deviennent familiers aux téléspectateurs. Par la voix, cela veut dire par ce qui est énoncé (vocabulaire, registres lexicaux, sens proposé...) et par la manière dont cela est énoncé (intonation, accent -du Sud-Ouest de préférence-, intensité, rythme...).

Les propos d'avant match ont une double fonction par rapport à la grammaire événementielle : d'une part, mettre en situation (lieu, temps, taille du public, ambiance, équipes, enjeux), d'autre part, donner le ton : «Dans quelques minutes va se jouer un match que bien des confrères ont qualifié de match du siècle, France/Afrique du Sud, et ils n'ont sans doute pas tort. Voici pourquoi. Les célèbres Springboks sont restés invaincus de 1906 à 1958, soit pendant plus d'un demi-siècle. 1958, année au cours de laquelle, le XV de France, commandé par Lucien Mias, alla les battre chez eux, le 16 août à Johannesburg par 9 points à 5. Et comme les Tricolores remportaient également peu après le Tournoi des Cinq Nations, on les considéra comme les véritables champions du monde de rugby. Cela, les Afrikanders n'ont jamais pu l'admettre, et pendant trois ans, ils ont préparé leur revanche. Et maintenant que j'ai situé pour vous l'importance de cette rencontre... » (F-AS/61) ; «Ça dépasse si vous voulez également les limites habituelles d'une finale car il s'agit d'une lutte un peu... j'allais dire d'une lutte fratricide, non pas fratricide mais fraternelle, entre une Préfecture et une Sous-préfecture, car Dax n'a jamais bien admis que ce soit Mont de Marsan qui soit la Préfecture des Landes. Ça va très loin dans le fond » (D-MdeM/63).

C'est par ces quelques phrases que le journaliste (Roger Couderc) introduit en les contextualisant les retransmissions d'un match international et d'une finale du championnat de France. Certes, l'importance de la rencontre est chaque fois mise en avant à travers un vocabulaire empruntant aux stéréotypes éculés du commentaire sportif (« match du siècle », « apothéose », « dépasse les limites d'une finale »), tandis que son intérêt pour les téléspectateurs est rappelé par la référence appuyée à une rivalité certaine mais volontairement exagérée. Cependant quand il s'agit d'une finale, le régionalisme, voire le campanilisme, remplace l'emphase patriotique et l'union sacrée devant l'étranger revanchard : l'enjeu local, avec

$16^{\text {ème }}$ homme du XV de France. Georges de Caunes qui l'a remplacé pour quelques rencontres après son éviction provisoire de l'ORTF en 1968 ne s'est jamais des «prises de pouvoir » de cette nature. 
toutes les animosités que l'on peut imaginer entre voisins, se substitue à la lutte pour la suprématie mondiale. D'ailleurs, le commentaire relaye et exhibe les éléments emblématiques, voire folkloriques, des cultures des protagonistes (Bonnet, 2007) : «Il y a maintenant un Toro de Fuego sur votre écran. C'est Dax qui le fait partir. Il y a également des échassiers, vous allez les voir dans quelques secondes. Ils sont là les échassiers, et ça c'est Mont-de-Marsan! » (D-MdeM/63). Bien que ramenée à des indices, la culture du Sud-Ouest demeure aisément identifiable non seulement pour les Landais, mais aussi pour l'ensemble des téléspectateurs français.

Deux décennies plus tard, alors que la télévision « commerciale de service public » a succédé à la télévision gaullienne, les artefacts subsistent mais sous une forme moins martelée. Si le commentaire de France-Nouvelle-Zélande (1986) s'éloigne, sans pour autant les renier, des accents patriotiques -il est toujours question d'une « guerre »-, il en appelle cependant à la continuité territoriale de la France, intronisant pour la circonstance Nantes «capitale du rugby », alors que l'Ovalie ${ }^{1}$, contrairement à la France, se caractérise par une organisation multipolaire. De la même manière, c'est moins le folklore qui est convoqué pour les finales de championnat, que la dimension festive et la convivialité, le facteur humain servant de dernier indice à la communauté : «Merci de nous donner l'antenne. Ici le Parc des Princes, cette finale du championnat de France entre Toulouse et Toulon est commencée depuis plus d'une minute maintenant. Quarante-cinq mille spectateurs dans un Parc des Princes bruyant, enfumé même par les pétards des supporters. Une ambiance de fête pour une finale qui s'annonce royale entre les deux meilleures équipes du championnat cette saison » (T-To/85). Pour un match international, si le commentaire assure que l'ambiance est au rendez-vous, la dimension festive est relativisée : «Et voilà les Français sous les hurlements de 50000 spectateurs, un Parc des Princes plein à craquer, et la Fédération nous a dit, qu'ils avaient refusé, écoutez-moi bien, 10000 places ! »(F-PG/83) ${ }^{2}$. Ainsi que le relèvent Katz et Dayan

1 Plus qu'une simple communauté de pratiques, du moins pour ses membres, l'Ovalie est « un pays à la fois imaginaire et réel dont on pourrait vérifier les attributs cardinaux : une communauté, une culture, un territoire, une histoire, une organisation politique » (Noaillac, 2007).

2 «Le Parc des Princes est comble pour cette belle fête du rugby. On aurait pu mettre au moins 30000 spectateurs de plus (...) C'est vous dire si l'affiche est très courue » (T-BJ/97). 
(1996, 81), «les caractéristiques soulignées par la télévision afin d'identifier l'événement portent autant sur la dramaturgie cérémonielle que sur les gestes qui y répondent du côté du public. Les réalisateurs illustrent ces réponses en braquant leurs projecteurs sur les spectateurs présents. Il s'agit pour eux de transmettre des comportements jugés exemplaires. Il s'agit aussi de souligner la nature communautaire de l'expérience, l'unanimité de l'adhésion aux valeurs et aux symboles célébrés. Il s'agit aussi de montrer, non seulement l'intensité des sentiments manifestés, mais la diversité dont elle émerge ».

Dans les années 1990 et 2000, alors que la télévision devient de plus en plus réflexive et donc très encline à parler d'elle-même, les propos d'avant match sont aussi l'occasion de mettre en valeur le dispositif télévisuel (nombre de caméras, performance technique de la réalisation, présence dans les gradins des responsables de la chaîne...), de faire référence autant aux téléspectateurs qu'aux spectateurs, et parfois de cabotiner. Il faut dire que depuis les années 1970 (matches internationaux) et 1980 (rencontres nationales), la prise d'antenne s'est effectuée plus tôt, rallongeant d'autant la séquence d'avant-match. Guy Lochard et Manuel Fernandez (2007) soulignent que cet allongement permet de mieux " rendre compte de la mobilisation identitaire qui s'effectue autour de la rencontre chez les spectateurs des deux camps et dans laquelle le média télévisuel joue un rôle déterminant $»$. Il autorise également le franchissement de certaines limites entre l'espace du dehors et l'espace du dedans et prépare l'incursion des caméras, d'abord jusqu'à la porte des vestiaires, ensuite en leur sein (avec Canal Plus). Mais il est surtout un des moyens dont s'est dotée la télévision pour médier l'événement, l'inscrire dans une socioculture plus vaste que celle de l'Ovalie, mais compatible avec elle. Ainsi, en l'amplifiant, le commentaire et les images lui donnent un écho de plus en plus large et le mettent en perspective avec d'autres événements, internes ou externes au monde du rugby : enjeux mondiaux du test match (F-AS/61), querelle interne au Département des Landes (D-MdeM/63), commémoration de la mort de joueurs (F-E/68), retraite de Roger Couderc (F-PG/83), élections législatives $(\mathrm{T}-\mathrm{BJ} / 97) \ldots$ 


\section{Publics, scènes du spectacle et dispositifs télévisuels}

Comme tous les sports de compétition, le rugby est aussi un spectacle et plus précisément un spectacle qui tire son principal intérêt de la dramaturgie propre aux sports collectifs de combat (Pociello, 1998), de son unicité, de son incertitude et des performances qu'il promet, mais aussi de son intertextualité : tout match s'inscrit à la fois dans une histoire et une mémoire et dans un avenir, autrement dit dans d'autres matches passés ou futurs qui généreront des discours de journalistes et de supporteurs et pour certains d'entre eux des grands récits et des légendes ${ }^{1}$. Il est d'abord un spectacle in praesentia, puisqu'il réunit dans un même lieu et au même moment des joueurs et des spectateurs, le terrain et les gradins étant mis à distance par une coupure matérielle (la main courante ou la grille) et par l'existence de deux univers de sens. Ensuite un spectacle télévisé, c'est-à-dire d'une part, un spectacle dans lequel un dispositif socio-technique instaure un système de représentations et de médiations entre l'événement et les téléspectateurs, d'autre part, un spectacle mis en scène par la télévision, pour la télévision, avec le formatage désormais bien connu que cela implique (Coulomb-Gully, 2001).

Depuis les années 1960, c'est incontestablement le terrain qui constitue la scène principale : il est par excellence l'espace du jeu, le lieu des acteurs et de l'action à partir duquel le match est montré, raconté et scénarisé. Les spectateurs y sont néanmoins présents, mais hors champ, à travers leurs cris, applaudissements, sifflets et chants, autant de manifestations sonores que les commentateurs signalent pendant le match, par exemple durant les arrêts de jeu, nombreux au rugby, ou lors de moments clés (points marqués, bagarre...).

Mais il existe d'autres scènes qui, pour être secondaires, ne participent pas moins au dispositif proposé aux téléspectateurs. Si le stade proprement dit est rapidement évacué par l'image et le commentaire

1 En outre, la spectacularisation se démultiplie bien au-delà du match retransmis puisque, pour s'en tenir à la seule télévision, le match peut être rediffusé sous le même format (par exemple par une chaîne thématique sportive comme ESPN) et, sous la forme d'un résumé ou de fragments, ressurgir dans des images d'avant-match (y compris avant la prise d'antenne proprement dite), dans un Journal Télévisé ou dans ces émissions de plateau qui fleurissent sur les chaînes généralistes et spécialisées. 
(le Parc des Princes est seulement «comble », le Stade de France « magnifique » et celui de Nantes «plein à craquer »), il n'en va pas de même des gradins et des tribunes. Spectateurs et supporteurs avec leurs démonstrations aussi voyantes ${ }^{1}$ que bruyantes et leur folklore font partie du spectacle télévisé, surtout quand ils « font couleur locale $»^{2}$ et/ou que les manifestations (encouragements, joie, colère...) traduisent un sentiment d'appartenance à la communauté des « regardants ». Par ailleurs, en les mentionnant, le commentaire en fait le relais indiciaire de l'opposition structurale du jeu et un élément non négligeable de la dramatisation des situations et des enjeux. A partir des années 2000, à côté des spectateurs traditionnels (mais beaucoup plus grimés et habillés aux couleurs des clubs qu'autrefois), la caméra et les commentaires s'attardent avec complaisance sur les publics nouveaux : supporteurs ultras aux banderoles plus nombreuses et plus exotiques que les anciennes, femmes (épouses de joueurs, « pom-pom girls » importées des Etats-Unis et d'autres sports, supportrices...), VIP invités par les sponsors ou les instances officielles du rugby...

Le spectacle réside aussi dans ce que le dispositif rajoute à l'écran et que le discours commente : « on voit ici, y'a des caméras partout ! Là elles sont même dans les vestiaires. Vous avez vu tout à l'heure la porte des visiteurs » (F-NZ/79) ; «Pendant ce temps, peut-être que Henri Carrier peut nous montrer le graphoscope, c'est une innovation dans ce reportage qui va nous montrer l'occupation du terrain par les deux équipes. Voilà, alors sur cet écran, c'est symptomatique : à gauche, vous voyez l'occupation toulousaine, oui, et à droite, l'occupation toulonnaise. On constate que, territorialement, Toulouse qui est menée trois à zéro par Toulon a nettement dominé » ( $\mathrm{T}-\mathrm{To} / 85)$. Alors que les procédés techniques se perfectionnent au fil des ans (replays, ralentis, loupes, incrustations, systèmes vidéo-informatiques...), les procédés rhétori-

1 «Alors que vous découvrez toujours des banderoles 'allez France', 'vive la France', 'allez l'Ecosse' » (E-F/68).

2 La retransmission d'Agen/Dax (1966), match pauvre en couleurs et en rivalité de clocher, ne fait guère référence aux spectateurs. Ainsi, à la mi-temps, la caméra se contente de filmer longuement la foule, tandis que les micros d'ambiance captent « passivement» les bruits de fond, le tout sans autre commentaire que « Je vous laisse regarder maintenant pendant cette mi-temps tous les croquis qu'Henri Carrier et ses caméras vont cueillir pour vous dans la foule, cette foule sans laquelle il n'y aurait pas de véritable ambiance, il n'y aurait pas de véritable finale du rugby ». 
ques ne sont pas en reste. Nous en évoquerons deux, particulièrement récurrents :

- l'interpellation du téléspectateur ${ }^{1}$, ce qui revient à lui montrer que l'on fait très attention à lui, voire qu'il est un ami ou encore mieux un partenaire certes imaginaire car hors co-présence, mais toujours imaginé. L'interpellation fait appel à des procédés bien connus qui relèvent du registre de la conversation (utilisation du je, vous, nous au détriment de la troisième personne, recherche systématique d'interactions...).

- l'ostentation avec laquelle les commentateurs insistent sur le dispositif, que leur parole soit un complément à caractère didactique de l'image (« à gauche, vous voyez l'occupation toulousaine, oui, et à droite, l'occupation toulonnaise ») ou un simple relais de celle-ci (« alors que l'on voit ici, y'a des caméras partout »). Alors qu'il était autrefois moins montré et parlé car il fallait, au nom de la transparence du direct, prouver que les choses se passaient comme on les montrait, le dispositif se manifeste désormais régulièrement dans l'image et le son, ce qui est entre autres choses le signe d'un changement de statut du téléspectateur.

C'est en fait au tournant des années 1970 que le commentateur cesse de traiter le téléspectateur comme un spectateur à distance (Lochard, Fernandez, 2007), évolution du dispositif oblige. En quelque sorte, le « mieux que si vous y étiez » remplace peu à peu le traditionnel «Imaginez que vous êtes assis sur les tribunes du stade de Colombes » (F-AS/1961). En effet, jusqu'à la fin des années 1960 le téléspectateur

1 «Alors que le coup d'envoi va être donné dans quelques secondes, c'est toujours le moment où la gorge du reporter se serre. Je sais que vous êtes des millions devant vos postes et c'est pourquoi, Pierre Sabbagh et moi-même espérons ne pas vous décevoir, espérons que les caméras et les commentaires seront dignes des deux équipes, du rugby et de votre enthousiasme » (F-AS/61) ; « Oh ! j'ai le cour à 140 à l'heure ! Boum boum boum boum boum boum boum ! il fait boum boum ! Et le vôtre aussi mes amis devant votre écran car il y a, c'est pas la peine de le nier, pourquoi le nier, c'est stupide, il y a des matches comme ça où le drame naît du score » (F-NZ/79) ; « Merci à Toulouse et à Toulon d'offrir un tel spectacle, quelque soit d'ailleurs le vainqueur car, vraiment, le spectacle est assuré dans cette finale suivie par quarante-cinq mille spectateurs et des millions de téléspectateurs qui nous font l'amitié de nous écouter et de nous regarder, sur Antenne 2 » (T-To/85). 
voit moins de choses que le commentateur à qui il est contraint de faire confiance : « D'où nous sommes placés, exactement au-dessus d'eux, nous les voyons venir ces hors jeu, nous les voyons avec Loÿs Van Lee. On les voit avant même que l'arbitre les siffle » (E-F/68)1. Onze ans plus tard, sa perception est meilleure que celle qu'il aurait eu s'il était dans les tribunes, ainsi que le souligne le commentaire qui affirme au passage la suprématie du spectacle télévisuel sur le spectacle vivant : "Voyez une caméra qui prend le contre-plan : c'est-à-dire que vous avez Wilson de face et vous voyez les poteaux si le ballon passe, vous le verrez aussi bien que l'arbitre de touche » (F-NZ/79). A la position transcendantale des commentateurs, correspond celle, tout aussi surplombante, des téléspectateurs qui voient ce que ni les spectateurs ni les arbitres voient.

La retransmission n'est donc plus l'équivalent d'une monstration au sens d'André Gaudreault, c'est-à-dire d'une transposition « réaliste » de ce qui se passe ici et maintenant sur le terrain ${ }^{2}$.

Cette évolution peut faire l'objet de trois grilles de lecture différentes, mais compatibles :

- une dissociation du spectacle vivant du spectacle télévisuel qui met en évidence que le rugby (ou le football, la politique, la religion...) à la télévision, c'est d'abord de la télévision (CoulombGully, 2001), étant entendu que ce que cette dernière donne à voir est pour une part lié aux relations qu'elle entretient avec la discipline sportive et ses acteurs. Cette dissociation est d'autant plus forte que les progrès de l'éducation télévisuelle des téléspectateurs rendent inutile l'artefact de substitution au spectateur pour éprouver émoi et partage de l'émotion sportive et qu'au fil des années, les téléspectateurs ont intégré des pratiques de réunion, voire de rassemblement, pour regarder les matches à la télévision ;

1 Le hors-jeu n'apparaît pas à l'écran.

2 Ainsi, par exemple, c'est parfois le replay ou le ralenti qui est choisi pour commenter, plutôt que l'action en temps réel : «Et voici le ralenti. Remise en jeu de Dalton à l'ouverture des Blacks (...) Je ne sais pas comment la balle est venue ici sur Donaldson qui ouvre. Regardez ici, vous allez voir » (F-NZ/79). 
- une évolution des régimes de mise en image (Lochard, 2007) et en son en relation avec les transformations des stratégies d'énonciation et des stratégies éditoriales : comme tout programme, la retransmission est la concrétisation d'un projet communicationnel mis en oeuvre à l'intention d'une cible prédéfinie en vertu d'une stratégie éditoriale de la chaîne (Lochard, 2005) et qui suppose un avant et un après match ;

- le signe d'un échec, du moins si on veut bien suivre Daniel Dayan et Elihu Katz $(1996,99)$ : «Quels que soient ses efforts, la télévision ne peut ni nous mener à l'événement, ni l'amener dans notre living room. Cet échec s'accompagne d'une offre de compensations. Il s'agit de dédommager des spectateurs qui n'auraient reçu qu'une expérience de seconde main en faisant ouvertement appel aux ressources du spectacle. Il s'agit d'offrir au spectateur un nouveau type d'expérience cérémonielle, expérience qui n'est accessible que de chez soi ; de remplacer le 'faites comme si vous y étiez' par la revendication du 'n'y être pas'». Dans cette perspective, on ajoutera que cette offre de compensations s'inscrit dans une logique de surenchère permanente. Ainsi, alors que le dispositif in vivo installe des écrans géants dans l'enceinte du stade, la télévision doit « en faire toujours plus » afin de contrebalancer cette sollicitation cognitive.

\section{Cérémonies télévisuelles et/ou fêtes du rugby ?}

Bien au-delà de la seule Ovalie, les retransmissions de matches de très haut niveau rassemblent une « diaspora » de téléspectateurs dispersés sur le territoire national et qui ont plus ou moins conscience d'appartenir à la communauté symbolique de ceux qui suivent à distance ces «cérémonies télévisuelles de confrontation» (Dayan, Katz, 1996), réactivant des sentiments d'appartenance non seulement au monde de ceux qui « aiment le sport », mais encore à la France par-delà ses divisions sociales, culturalo-territoriales, ethniques et sexuées. La télévision met en scènes avec force moyens et effets d'annonce ces rassemblements gorgés de sens sous la forme de grandes messes au cours desquelles la célébration de l'événement sportif est toujours l'occasion d'autres célébrations plus essentielles pour la communauté nationale.

Nous avons évoqué supra le traitement différencié des finales de championnat et des matches internationaux. En effet, il ne nous semble 
pas que les finales, telles qu'elles sont données à voir et à entendre par la télévision, répondent totalement à la grammaire des cérémonies télévisuelles. Si elles sont bien des événements, font l'objet d'une planification, sont précédées d'une communication massive destinée à produire de l'attente, sont retransmises en direct, sont soumises à un protocole et un rituel plus ou moins lourd et produisent à la réception un sentiment d'appartenance communautaire (le ressenti de chacun est voisin du ressenti des autres), elles ne rassemblent pas tout un pays devant les écrans, tant quantitativement (les parts d'audience sont éloquentes sur ce point), que qualitativement. Car l'événement n'est pas «massif» au sens où l'entend Gérard Derèze $(1998,33)$ par référence à la «masse de l'événement » définie par Abraham Moles (1972, 91) : "On définira la masse d'un événement (...) comme produit du nombre de personnes qui sont affectées dans leur sphère personnelle par le degré d'affection [ou de participation ou d'émotion] qu'elles en subissent $»$. Certes, le commentaire parle toujours de foule, de stade archi-comble -chiffres arrondis avantageusement à l'appui-, de billets supplémentaires que l'on aurait pu vendre, puis fait immanquablement référence aux « millions de téléspectateurs » et plus récemment aux téléspectateurs massés devant des écrans géants dans les deux villes concernées, d'autant que désormais l'image les montre. Mais nous sommes ici davantage en présence d'une routine journalistique et de stéréotypes discursifs que d'une rhétorique spécifique aux cérémonies. Quant au sentiment d'appartenance, force est de constater qu'il n'est pas national comme dans les matches internationaux : « on a gagné », crient de manière fusionnelle ${ }^{1}$ ceux qui supportent l'équipe victorieuse, «ils ont gagné » disent ou pensent de manière distanciée les spectateurs et les téléspectateurs « neutres », très largement majoritaires.

Cependant, si elle est moindre, la masse de l'événement n'est pas pour autant absente du commentaire car l'émotion, la convivialité et plus spécialement la dimension festive sont mises en avant lors des finales de championnat, alors qu'elles sont relativisées -les deux dernières surtout- dans les matches internationaux ${ }^{2}:$ « c'est une véri-

1 «Le caractère fusionnel de ce sentiment est démultiplié par les mouvements de rassemblement qui s'actualisent lors des manifestations de rue faisant suite au match (Calbo, 2000, 401).

2 Les termes « fête » et «événement» ne sont pas interchangeables dans la syntaxe du commentaire sportif. En effet, ils charrient des imaginaires, des discours et des 
table fête folklorique landaise avec des centaines et des centaines de landais (...) Il sent la poudre, ce match, cette finale qui sent la poudre au propre comme au figuré, car véritablement jamais de ma vie dans une finale je n'ai vu une atmosphère aussi formidable (...), et vous voyez cette véritable fête du rugby, ces gerbes d'étincelles, toutes ces danses, tous ces orchestres et ces banderoles, en un mot, tout ce qui représente et tout ce qui fait la grandeur et la beauté de la finale du rugby, qui je le répète, est la grande fête du rugby français » (D-MdM/63). Au-delà même de l'évident stéréotype discursif et performatif que l'on retrouve dans tous les « avant-match » des finales du corpus ${ }^{1}$, c'est une forme de topos au sens rhétorique du terme dans la présentation du match qui est donnée à entendre.

Le commentaire rappeler régulièrement que la fête est un jour particulier qui rompt avec l'ordinaire des compétitions et durant lequel se déroulent des manifestations spécifiques : le match stricto sensu avec sa pompe et son rituel (présentation des équipes au Président de la République, échange de fanions entre les deux capitaines...) et des micro-événements organisés ou tolérés (toro de fuego, échassiers, bandas, pétards...). La fête est aussi un rassemblement massif, générateur d'exaltation (Durkheim, 1912) et plus précisément un acte collectif, au sens où tout est fait pour que le groupe participe activement (le thème de la communion est surexploité par les commentateurs) et que l'événement se déroule dans des formes suffisamment spécifiques pour qu'il ne s'agisse pas d'un simple rassemblement : convivialité ${ }^{2}$, liesse, enthousiasme et bien, sûr, débordements : «Il y a du monde vraiment sur la pelouse, au-delà même peut être des limites possibles. Nous sommes nous-mêmes très près de la ligne de touche, environ à un mètre et souhaitons qu'il n'y ait pas de débordements trop percutants. Car vous voyez, les gens, au fur et à mesure que je vous parle d'ailleurs, vous voyez les gens sont vraiment très très près, tassés près de la ligne de touche, et cela me fait tout de même un peu peur » (D-MdM/63);

pratiques qui, s'ils sont médiatisés par la télévision et pour la télévision, ne relèvent pas de la même grammaire médiatique.

1 Et même lors de la transmission d'antenne : en 1977, Léon Zitrone qui vient de commenter la course du Tiercé passe l'antenne pour la retransmission de BéziersPerpignan en disant « Les chevaux et l'arrivée s'effacent pour le rugby, c'est la grande fête du rugby, à vous messieurs les rugbymen » .

2 «Il n'y a plus d'Agenais ou de Dacquois ici, il n'y a plus que des amoureux du rugby » (D-B/66). 
« Alors que vous continuez à regarder ces images vraiment étonnantes et de tous les cotés maintenant, ça va être difficile pour les faire sortir, ils sont absolument déchaînés » (idem) ${ }^{1}$.

Mais cette fête, et c'est en cela qu'elle se distingue des dimensions festives présentes dans certaines grandes cérémonies évoquées par Daniel Dayan et Elihu Katz, est fondamentalement, d'une part, celle des territoires ${ }^{2}$ dont les équipes défendent au sens propre comme figuré les couleurs et, d'autre part, celle du rugby, c'est-à-dire d'une discipline sportive $^{3}$ et de ses terroirs ${ }^{4}$, cette « terre de rugby » surtout effective dans la moitié sud de la France. Ainsi, Dax-Mont de Marsan (1963) est annoncé comme « l'apothéose du rugby landais », tandis que BéziersPerpignan (1977) est l'occasion, outre les vieilles rivalités de voisinage, d'évoquer pêle-mêle la corrida, la vigne, les langues locales, les lieux symboliques des deux villes (allées Paul Riquet pour Béziers, Castillet pour Perpignan)... Ces éléments contextuels sont essentiels, car tout autant que les artefacts visuels et auditifs (drapeaux, écharpes, bandas, slogans en langues locales...) ils permettent aux commentateurs de qualifier ces finales de « festives ».

1 On soulignera que le recours discursif à la fête (souvent modalisée en fête folklorique), très présent dans les années 1960 et 1970, s'estompe à partir des années 1980, peutêtre parce que les dimensions festivo-territoriales ont perdu en épaisseur sociale et symbolique ce qu'elles ont gagné en visibilité immédiate (forêt de drapeaux dans les tribunes, exhibition massive et ostentatoire des produits dérivés vendus dans les boutiques des clubs : écharpes, casquettes, tee-shirts...). Ou que les aspects formels rituels et très organisés de la fête ont peu à peu pris le pas sur les dimensions moins encadrées et préparées, voire spontanées. Ou bien que l'insistance des organisateurs et des médias sur les « spectateurs de marque » car le stade de rugby est devenu un lieu ou l'on va pour être vu, a pour contrepartie une plus grande désaffection pour le public ordinaire.

2 Pour Daniel Dayan et Elihu Katz, les cérémonies télévisuelles sont remodelées afin de relativiser fortement leur inscription territoriale.

3 « Répétons-le, une finale avant tout, c'est la grande fête du rugby » (D-B/66) ; « Incontestablement, tout au long de la saison, le Stade Toulousain a dominé cette année rugbystique, suivi de très près d'ailleurs par le Racing Club Toulonnais. Donc, ça n'est une surprise pour personne des gens du rugby de retrouver aujourd'hui Toulon et Toulouse qui s'affrontent » (T-To/85); « Oui, effectivement c'est la grande fête, après tant de matches au niveau des poules » $(\mathrm{T}-\mathrm{Br} / 96)$.

4 Andy Smith (2001) démontre l'importance de la dimension territoriale pour les amateurs de rugby, ce qui les conduit à s'intéresser de manière privilégiée, in situ ou par médias interposés, aux clubs proches de leur lieu de vie, mais aussi au XV de France en raison de son rapport au rugby des terroirs. 
En même temps, l'invocation de la fête et de la «qualité de l'affiche » est une double promesse : celle de la participation et de la convivialité, et celle du « beau rugby » et du « combat». La fête (ou son illusion) agit comme garant de l'enjeu et de la qualité du match, tandis que la qualité de l'affiche est un gage de la dimension festive. Cette invocation est un artefact discursif destiné à éveiller l'intérêt des téléspectateurs ne supportant aucune des deux équipes, qu'ils soient des aficionados avérés ou qu'ils appartiennent à un public plus large intéressé par la dimension sportive, voire simplement événementielle de la finale. C'est donc un moyen de compenser la moindre participation émotionnelle des téléspectateurs français inhérente à ce type de compétition, amoindrissement d'autant plus marqué que, contrairement aux matches internationaux, le commentateur ne peut ouvertement prendre parti pour l'une ou l'autre équipe. Pour quel résultat ? C'est à une étude de réception qu'il appartient de répondre. En son absence, on fera une double hypothèse : d'une part, l'immersion dans la communauté des téléspectateurs d'individus et de groupes spatialement et culturellement dispersés dépend largement de leur engagement dans l'événement social et médiatique ; d'autre part, le mode de participation «intellectuel » et « corporel » de chaque téléspectateur est aussi fonction du lieu et des conditions sociales de réception : la solitude dans un salon renvoie à une situation sociale différente de l'immersion dans un lieu public, café ou place équipée d'un écran géant.

La retransmission d'un match par la télévision a une double nature qui n'est pas sans incidence sur sa réception : elle est, par certains côtés, l'image plus ou moins déformée -et aujourd'hui plus que moins, scénarisation oblige- d'un spectacle in praesentia qui puise sa raison et son mode d'être dans un système d'organisation propre au sport ainsi que dans une culture dépassant largement le cadre sportif, et par d'autres, un spectacle construit par un dispositif socio-technique complexe et qui possède sa temporalité, sa logique, ses enjeux, ses stratégies symboliques et socio-économiques. Mais incontestablement, c'est la deuxième dimension qui domine, en tout cas pour les téléspectateurs; le spectacle donné à voir et à entendre sur le « petit écran », ou si l'on préfère médié par la télévision, est bien autonome par rapport au spectacle premier, il est d'abord de la télévision. Plutôt que de conclure 
à la victoire probable, voire déjà acquise, de la culture d'écran sur la culture du stade, on préfèrera y voir, avec Georges Vigarello (1998), une nouvelle façon de regarder les matches susceptible de développer de nouvelles compétences et, par rapport à la discipline sportive qui nous préoccupe ici, de nouvelles manières d'être au rugby.

\section{Références bibliographiques}

Barthes R., Mythologies, 1956, Paris : Le Seuil.

Bonnet V., 2007, « Rugby, médias et territoire », Mots - Les Langages du Politique, $\mathrm{n}^{\circ} 84$, Politiquement sportif, juillet, 35-49.

Bonnet V., Boure R., Cassagne J.-M., 2007, « Les valeurs rugbystiques dans les commentaires médiatiques », Sciences de la Société, n72, octobre, Sport et médias, $56-75$.

Bonnet V., Boure R., 2008, « Le rugby entre patrimoine, récit et communication. Le cas de Midi Olympique », Semen, n²6, 143-159.

Boure R., Cassagne J.-M., 2007a, «Le rugby est-il soluble dans ses valeurs ? », colloque Rugby, médias, éducation et transmission des valeurs, Universités Montpellier 1 et Montpellier 3, Béziers, 13-15 juin, Actes à paraître.

Boure R., Cassagne J.-M., 2007b, « Les mots des matches ou les territoires discursifs du rugby », in Cahuzac H., Lochard G., dirs., L'ovale dans la lucarne. Le rugby à la radio et à la télévision, Bruxelles/Paris : De Boeck/INA, 177-192.

Calbo S., 2000, « La production de l'appartenance nationale. Le cas de la Coupe du Monde 1999 », in Gabaston P., Leconte B., Sports et télévision. Regards croisés, Paris : L'Harmattan, coll. Communication et civilisation, 389-405.

Chion M., 1990, L'audio-vision, Paris : Nathan, coll. Nathan Université.

Coulomb-Gully M., 2001, La démocratie mise en scènes. Télévision et élections, Paris : CNRS Éditions, coll. CNRS Communication.

Darbon S., dir., 1999, Rugby d'ici. Une manière d'être au monde, Autrement, $\mathrm{n}^{\circ} 183$.

Dayan D., Katz E., 1992, Media events. The live broadcasting of history, Cambridge, MA : Harvard University Press.

Dayan D., Katz E., 1996, La télévision cérémonielle, Paris : PUF.

Derèze G., 1998, « «De la médiatisation des grandes compétitions sportives », Communications, ${ }^{\circ} 67,33-42$.

Durkheim E., 1998 [1912], Les formes élémentaires de la vie religieuse, Paris : PUF, col. Quadrige.

Fernandez M., 2004, « L'évolution du commentaire sportif. De l'épopée à analyse rationnelle », Médiamorphoses, $\mathrm{n}^{\circ} 11$, Le sport médiatisé. Du voir au savoir, 57-61.

Fernandez M., Lochard G., 2007, « Roger Couderc ou l'histoire d'un conflit de légitimité : les mises en scène télévisuelles françaises dans les rencontres internationales de 1961 à 1983 », in Cahuzac H., Lochard G., dirs., L'ovale dans la lucarne. Le rugby à la radio et à la télévision Bruxelles/Paris : De Boeck/INA, 111-138.

Gaudreault A., 1988, Du littéraire au filmique. Système du récit, Paris : Klincksieck.

Lochard G., 2007, «Les régimes de visualisation des compétitions sportives », Sciences 
de la Société, $\mathrm{n}^{\circ} 72$, octobre, Sport et médias, 8-25.

Lochard G., 2005, L'information télévisée. Mutations professionnelles et enjeux citoyens, Paris, CLEMI/INA/Vuibert.

Moles A., 1972, " Notes pour une typologie des événements », Communications, $\mathrm{n}^{\circ} 18$.

Noaillac F., 2007, «Un magazine communautaire, Rencontres à $X V »$, in Cahuzac H., Lochard G., dirs., L'ovale dans la lucarne. Le rugby à la radio et à la télévision Bruxelles/Paris : De Boeck/INA, 111-138.

Pociello R., 1998, «Sur la dramaturgie des jeux de combat », Communications, n67, 149-164.

Smith A., 2001, La passion du sport. Le football, le rugby et les appartenances en Europe, Rennes : PUR.

Veron E., 1981, Construire l'événement. Les médias et l'accident de Three Miles Island, Paris : Éditions de Minuit.

Véron E., 1983, « Il est là, je le vois, il me parle », Communications, n³8, 98-121.

Vigarello G., 1998, «Le marathon entre bitume et écran : les métamorphoses d'un spectacle », Communications, $n^{\circ} 67,211-215$. 